\title{
Traffic Control Scheme for Social Optimum Traffic Assignment with Dynamic Route Pricing for Automated Vehicles
}

\author{
Qiong Lư ${ }^{1 *}$, Tamás Tettamanti ${ }^{1}$ \\ ${ }^{1}$ Department of Control for Transportation and Vehicle Systems, Faculty of Transportation Engineering and Vehicle Engineering, \\ Budapest University of Technology and Economics, H-1111 Budapest, Stoczek street 2., Hungary \\ * Corresponding author, e-mail: qiong.lu@edu.bme.hu
}

Received: 19 May 2021, Accepted: 15 June 2021, Published online: 30 August 2021

\begin{abstract}
In transportation modeling, after defining a road network and its origin-destination (OD) matrix, the next important question is how to assign traffic among OD-pairs. Nowadays, advanced traveler information systems (ATIS) make it possible to realize the user equilibrium solution. Simultaneously, with the advent of the Cooperative Intelligent Transport Systems (C-ITS), it is possible to solve the traffic assignment problem in a system optimum way. As a potential traffic assignment method in the future transportation system for automated cars, the deterministic system optimum (DSO) is modeled and simulated to investigate the potential changes it may bring to the existing traditional traffic system. In this paper, stochastic user equilibrium (SUE) is used to simulate the conventional traffic assignment method. This work concluded that DSO has considerable advantages in reducing trip duration, time loss, waiting time, and departure delay under the same travel demand. What is more, the SUE traffic assignment has a more dispersed vehicle density distribution. Moreover, DSO traffic assignment helps the maximum vehicle density of each alternative path arrive almost simultaneously. Furthermore, DSO can significantly reduce or avoid the occurrence of excessive congestion.
\end{abstract}

\section{Keywords}

traffic assignment, stochastic user equilibrium, deterministic system optimum, social optimum traffic assignment, utility, automated vehicles

\section{Introduction}

Traffic assignment is a process to allocate a given set of travelers to a specific transport system. As part of the transportation planning process, one of the purposes of traffic assignment is to assess the impact of limited improvements and extensions on a specific transportation network by assigning estimated upcoming trips (Patriksson, 2015). The traffic assignment model must recognize the individual traveler's route choice behavior. There are two different route choice models: user equilibrium (UE) and system optimum (SO) traffic assignment (Pauer and Török, 2019; Seger and Kisgyörgy, 2020). Wardrop (1952) proposed these two fundamental principles to model the travelers' route choice. The UE principle means the travelers minimize their own travel costs when they travel to their destination. In this case, all the used routes have the same travel costs between a particular origin-destination (OD) pair. No driver could reduce journey cost by altering to another path. Although many factors affect the drivers' route choice, travel time is the chief element in travel cost.
So, travel cost and travel time are used interchangeably in this paper. The SO means that the average journey time is at a minimum. By affecting the drivers' route choice may lead travelers to minimize the total travel time. All the paths between a specific OD pair share the same marginal travel times (Wardrop, 1952). Pigou (2013) found that the UE has generally not minimized the total travel cost. Centralized control over route choice and congestion pricing strategy are two possible methods that can be used to obtain a SO assignment (Patriksson, 2015).

With the rapid development of technology, more and more communication equipment has been introduced into the transportation system. The Collaborative Intelligent Transport System (C-ITS) uses Vehicle-toEverything (V2X) communication techniques to improve road safety and traffic efficiency (Sjoberg, et al., 2017). This technology can work as a central controller providing a probability to control the vehicle's route choice in the routing phase. Besides, the emergence of robo-taxi and the 
popularity of mobility-as-a-service may bring the direct central control into reality, i.e. the system-optimal flows can be obtained. Another way to realize the system optimum is to apply a congestion pricing strategy. It is the passenger's failure to notice the total cost they add that causes the difference between user optimum and system optimum. Any additional passenger of a network is a marginal user. To achieve system optimum, the passengers must be aware of the total cost they put on others. By applying a congestion pricing strategy, every trip-maker can realize the marginal cost caused by them (Beckmann et al., 1956).

Despite its perfect theoretical basis, the first best pricing scheme is of little practical interest and may not be valid at present. It is impractical to charge users on each network link. The first best pricing may distort the traffic allocation over the entire network and may cause degradation instead of improvement in social welfare in reality. In fact, drivers may not always have perfect information about the travel cost. Besides, they may not always make a reasonable decision and choose the route by their minds rather than travel time. When faced with a choice situation, the preferences of a person towards each alternative can be determined by the utility, reflecting each alternative's attractiveness. The utility is a function of the alternatives' attributes and the features of the decision-maker. However, the utility cannot be explicitly observed because it cannot quantify the characteristics influencing a person's decision. The utility is then modeled as being random. Consequently, the choice offers only the likelihood of which options are chosen (Sheffi, 1985).

As a potential traffic assignment method for future transport with automated vehicles, the deterministic system optimum (DSO) is modeled and simulated to investigate the potential changes it may bring to the existing traditional traffic system. In this paper, stochastic user equilibrium (SUE) is used to simulate the conventional traffic assignment method. By comparing the two simulation results, one can see the difference between the two traffic allocation modes. DSO has considerable advantages in reducing trip duration, time loss, waiting time, and departure delay under the same travel demand. The SUE traffic assignment has a more dispersed vehicle density distribution. Moreover, DSO traffic assignment helps the maximum vehicle density of each alternative path arrive almost simultaneously. Furthermore, DSO can significantly reduce or avoid the occurrence of excessive congestion.

This paper contains five sections. Section 1 introduces the background and the investigated problem. The travel time model, pricing scheme, and route choice model are inspected in Section 2. Section 3 clarifies the road network, travel demands, and the reroute algorithm. The trip information and traffic distribution are analyzed in Section 4. The conclusion is described in Section 5.

\section{Methodology}

This study investigates the potential benefits of the DSO traffic assignment over the SUE. Congestion pricing is an indirect traffic control method to realize the SO.

\subsection{Volume delay function}

Travel time models represent the temporal variations of link traffic flows and link travel times in dynamic traffic assignment formulations. To ensure the first in first out (FIFO) queuing discipline, Daganzo (1995) suggested that travel time models should only be a function of the amount of link traffic.

The four-step model has been extensively used as a travel demand forecasting model for decades. As a critical element in the travel demand forecasting process, traffic assignment allocates travel demands to the transportation supply (network) based on travel costs. The travel cost is usually considered to be the travel time between the origin and the destination. The Volume Delay Function (VDF) is commonly applied in static macroscopic traffic assignment to describe the resulting link travel times as a function of flow (the result of assignment), capacity, and free-flow travel time (constant parameters of the link). This function aims to reproduce congestion effects in the macroscopic model and serves as an objective function in the assignment problem where the travel times are minimized (Fukushima, 1984).

The US Bureau of Public Roads (BPR) developed a VDF, as shown in Eq. (1), in 1964, which is a well-known formula to determine the travel time in each utilized link (United State, Bureau of Public Roads, 1964).

$$
T(v)=T_{0} \cdot\left(1+\alpha \cdot\left(\frac{v}{c}\right)^{\beta}\right),
$$

where $T$ is the travel time (minute). $T_{0}$ is the free flow travel time (minute); $v$ is traffic volume [Passenger Car Unit $(\mathrm{PCU}) /$ hour]; $c$ equals the practical capacity (PCU/hour); $\alpha, \beta$ are dimensionless tuning parameters.

Unlike the traffic flow's physical representations, the VDF allows the flow to exceed the link capacity. In VDF, the $\mathrm{v} / \mathrm{c}$ ratio can be larger than 1 , which is impossible by considering the definition of road capacity. Consequently, the flow volumes applied in the macroscopic assignment 
are not rigidly referred to as the physically measured flows. The flow used in the VDF is treated more like a demand flow, which becomes delayed if it exceeds capacity. As a result, the VDF cannot be evaluated directly from the actual field measurements. Kucharski and Drabicki (2017) proposed a technique to overcome this problem by replacing the traffic flow with the traffic density (PCU/ km). They estimate the VDF by using density instead of flow. The formula is then straightforwardly written as:

$T(k)=T_{0} \cdot\left(1+\alpha \cdot\left(\frac{k}{k_{\text {crit }}}\right)^{\beta}\right)$.

Here, $k$ is the traffic density. $k_{\text {crit }}$ is the critical density at which the maximum flow occurs. $\alpha$ and $\beta$ are tuning parameters, similarly as used in Eq. (1).

\subsection{Pricing scheme}

The difference between UE and SO is accounted for by the individual user's failure to share the cost he/she contributes to the total travel cost. The theoretical background of congestion pricing relies upon the marginal-cost pricing (or the first best pricing) principle, which states that a toll equals the difference between the marginal social cost and the private cost to optimize the social surplus. The marginal travel cost of a link $a$ at the flow $T_{a}$ is defined as the increase in total travel cost on the link $a$ caused by an additional traveler. The difference between private and social cost is $T_{a}^{\prime}\left(k_{a}\right) \cdot k_{a} \cdot l_{a}$ (Patriksson, 2015). In order to achieve economic efficiency, every traveler must be made aware of the costs he/she imposes on other travelers. In this way, the traveler is supplied with an incentive to minimize social costs. Any SO problem may be solved as a UE problem by redefining travel costs as $C_{a}=T_{a}+T_{a}^{\prime}\left(k_{a}\right) \cdot k_{a} \cdot l_{a}$ (Patriksson, 2015).

The first derivative of the travel time model (Eq. (2)) is:

$$
\frac{d T}{d k}=\frac{T_{0} \alpha \beta}{k_{\text {crit }}}\left(\frac{k}{k_{\text {crit }}}\right)^{\beta-1} \text {. }
$$

Thus, the SO marginal cost pricing becomes:

$$
P(k)=k \cdot l \cdot \frac{d T}{d k}=\alpha \beta l T_{0}\left(\frac{k}{k_{\text {crit }}}\right)^{\beta} \text {. }
$$

Here, $T(k)$ is the travel time, which is the user equilibrium pricing. $k \cdot l \cdot \frac{d T}{d k}$ is the marginal travel time that the inserted vehicle has incurred. $l$ is the length of the road.

In this work, DSO assigned vehicles according to the pricing and travel time. On the contrary, the SUE allocated cars only considering the travel time.

\subsection{Route choice models}

When faced with a choice, an individual's preferences toward each alternative can be described by the utility, representing each alternative's attractiveness. The utility is a function of the attributes of the options and the decision maker's socioeconomic characteristics. However, the utility cannot be observed directly because the features that influence the individual's decision cannot be measured. Therefore, the utility is modeled as random. Consequently, the choice only provides the probability with which options are selected.

In Simulation of Urban MObility (SUMO), the default route choice algorithm is called Gawron (Lopez et al., 2018). For each driver, the Gawron algorithm calculates possibilities for selecting from a group of optional links. To compute these probabilities, the edge travel time of the previous simulation stage, the sum of edge travel times of alternative routes, and the prior likelihood of selecting a route are considered. The default route choice algorithm is applied to simulate the SUE. The route choice model of DSO is deterministic, which is realized via SUMO Traffic Control Interface (TraCI) (Lopez et al., 2018). In the simulation, DSO traffic assignment allocated all vehicles to the route with the lowest marginal travel time.

\section{Simulation scenarios}

To study the possible improvement of DSO assignment on the current traffic system, SUMO was utilized to simulate two scenarios with the same traffic demand on one network. A stochastic UE assignment was used to simulate the existing traffic system. The possible future DSO assignment was realized with the marginal cost pricing scheme.

\subsection{Transportation road network and demands}

As shown in Fig. 1, an artificial road network with three alternative roads was used to verify the proposed dynamic link-based marginal cost pricing strategy's validity. There was one pair of origin and destination. The origin edge was 400 meters long. At the end of it is 100 meters of connecting road. Three alternative routes converged the connection edge and their joint ends. At this intersection, a traffic signal light was equipped to regulate the traffic. The intersection was connected to the destination with an edge. At the start point of the destination, there was an intersection where another traffic light (traffic light 2) was located. A dummy edge was introduced to equip the traffic light. A variable speed signal (VSS) was applied to the destination. Traffic light 2 and the VSS were used to simulate the 


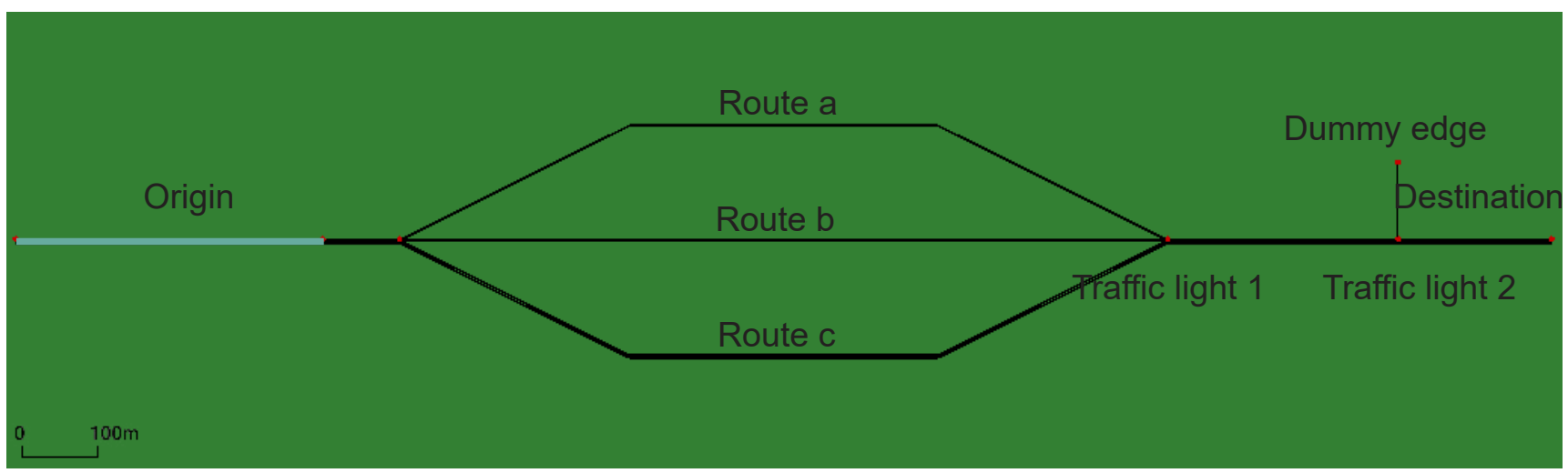

Fig. 1 Simulation traffic network

downstream traffic conditions. Except for route $a$, route $b$, and the dummy route, all edges have two lanes. The parameters of the three alternatives were listed in Table 1. Length means the length of the alternative routes without the common roads. $\alpha$ and $\beta$ were fitted by the least square method. The critical densities $k_{\text {crit }}$ were estimated from the fundamental diagrams of each route.

The travel time curves and pricing curves are shown in Fig. 2. The dashed curves are the travel time curves of available paths. The solid lines are the marginal pricing curves. As shown from the figure, when the vehicle density on the road is low, the curves are relatively flat. With the increase of density, the rate of increase of toll curve is obviously higher than the increment of travel time curve. This is because under the SO pricing scheme, the drivers would feel not only their own travel time, but also the marginal increment in travel time they would impose on

Table 1 Attributes of alternative routes

\begin{tabular}{lccc}
\hline & Route $a$ & Route $b$ & Route $c$ \\
\hline Length $(\mathrm{m})$ & 1071 & 1000 & 1071 \\
$\alpha$ & 0.95 & 1.12 & 1.91 \\
$\beta$ & 2.08 & 1.68 & 1.55 \\
$k_{\text {crit }}(\mathrm{veh} / \mathrm{km})$ & 50 & 50 & 70 \\
Lanes & 1 & 1 & 2 \\
\hline
\end{tabular}

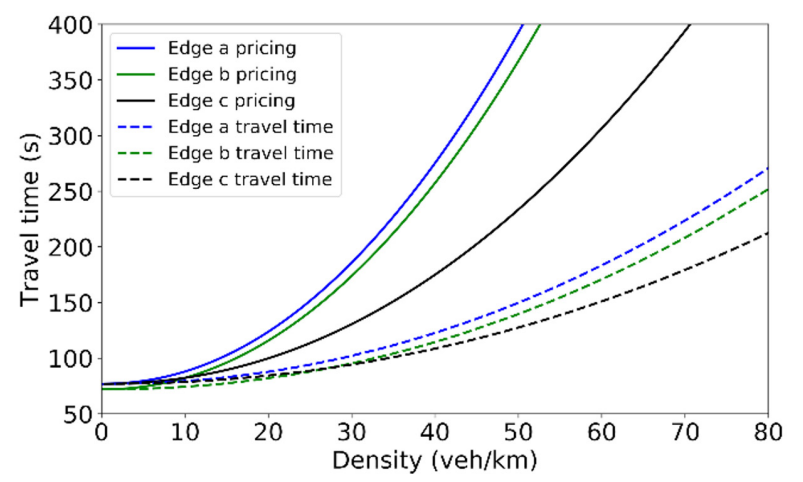

Fig. 2 Travel time and pricing curves other travelers along the same road. The pricing curves are more diffuse than the travel time curves. In the case of the same travel time, for example, 200s, the difference in the pricing curve is even more significant. This means drivers are more aware of the different capacities of alternative routes. More drivers will be directed to high-capacity roads to give full play to their carrying capacity.

As shown in Fig. 3, the travel demands for the simulation first increased gradually over time and then decreased gradually at the same pace when it reached its peak.

Vehicles could be routed dynamically in the ongoing SUMO simulation by enabling the cars to re-compute their route periodically. The routing procedure takes into consideration the current and the prior traffic situation. Therefore, it can adapt to congestion and other changes in the road networks. If the dynamic rerouting is applied to a simulation, the road network's travel times are collected for all edges. The collected edge travel times are updated periodically. When a vehicle is making a routing decision, it always takes the swiftest route to its destination according to travel times on different links. By properly defining the rerouting parameters, one can easily realize the user's optimum traffic assignment.

The SUMO routing algorithms aim to minimize the travel time between passengers' origin and destination by default.

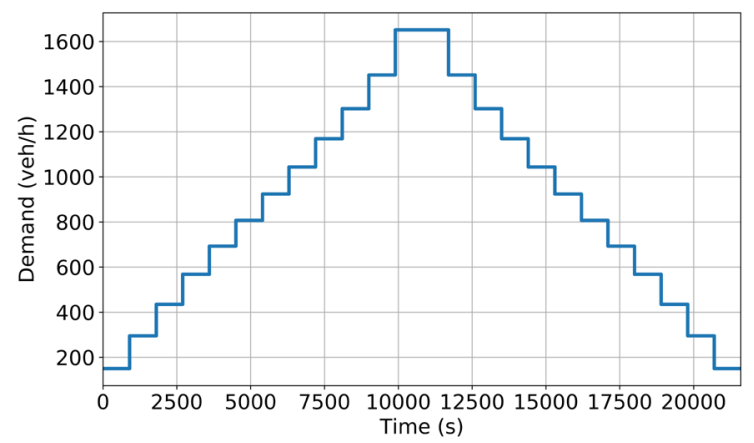

Fig. 3 Travel demands 
The travel time can either be obtained from the speed limits and edges' length, retrieved during the simulation runtime from the simulation state, or loaded from a data file. In this work, the Dijkstra routing algorithm was applied, and the travel times were retrieved from the simulation state.

\subsection{Rerouting algorithm}

In SUMO simulation, vehicles can have automatic routing in four ways: configuring options, incomplete trips or flows, a declaration in vehicle parameters, and using TraCI. In this paper, all vehicles were defined with incomplete trips. Consequently, all cars got automatically routed at insertion. Besides routing at departure, the configuring options were applied to realize the user's optimum traffic assignment. The configuring options were defined as follows. The rerouting probability equaled 1 to enable all vehicles in the network to re-compute their routes. The interval for updating the edge weights and the rerouting period was set to 2 seconds to maintain consistency with the system optimum traffic assignment setting. The importance of earlier edge weights for exponential averaging the edges' travel time was zero (as by default) to merely consider the current travel times.

By contrast, the optimized simulation rerouted vehicles with the help of the TraCI. As shown in Fig. 4, in TraCI, the travel time is changed according to the pricing method to let drivers feel the marginal travel time increment they put on other vehicles driving on the same road. At the start of the simulation, a counter $i$ was set to be 0 . The simulation ran by itself for two seconds. After which, the number of vehicles on each alternative road was retrieved to calculate the marginal travel time through the marginal pricing scheme. In the next stage, the marginal travel times for each route were sent to vehicles still on their way out, and these vehicles began to recalculate their routes based on the marginal travel time. The next step was to determine whether the counter $i$ was greater than 12600. If not, it was increased by one to the next iteration until it was greater than 12600 and the simulation stopped. The whole simulation process lasted 7 hours.

\section{Results}

During the simulation, trip information and the vehicle numbers on the alternative routes were collected.

\subsection{Trip information}

Each simulation has completed 5250 trips. The same parameters for each journey of the two simulations are

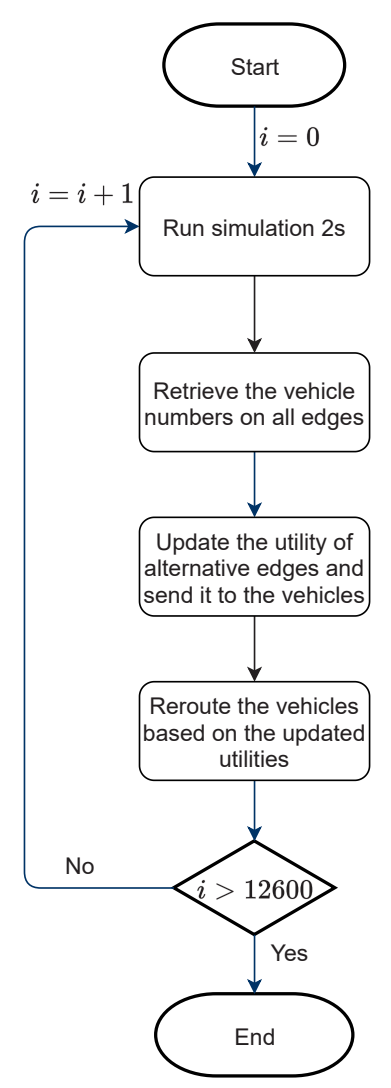

Fig. 4 Reroute algorithm

aggregated in the following table. In Table 2, five attributes of trips are listed. Trip duration is the time the vehicles needed to accomplish the route. Compared with the stochastic user equilibrium, the cumulative trip duration of the deterministic system optimum is reduced by $14 \%$. Route length stands for the aggregated length of vehicles' route. The two assignment methods are almost equal on this parameter. As a result of driving below the optimum speed, time loss occurs. This takes into account the slowdowns due to the intersections or congestions, etc. SO assignment loses significantly less time than UE. Around $26 \%$ of the time can be saved with the help of SO in the simulation scenarios. The waiting time is the time at which the speed of the vehicle is not faster than $0.1 \mathrm{~m} / \mathrm{s}$. The difference in waiting time is $17 \%$ between UE and SO

Table 2 The aggregated trip information of two methods

\begin{tabular}{lccc}
\hline Parameters & UE & SO & $\begin{array}{c}\text { Relative } \\
\text { change }\end{array}$ \\
\hline Trip duration (s) & 1834500 & 1575653 & $-14 \%$ \\
Route length (m) & 10739200 & 10723800 & $-0.1 \%$ \\
Time loss (s) & 991799 & 733784 & $-26 \%$ \\
Waiting time (s) & 492734 & 410589 & $-17 \%$ \\
Departure delay (s) & 78970 & 35284 & $-55 \%$ \\
\hline
\end{tabular}


assignment. The departure delay is the time the drivers have to wait until their journey starts. When there are too many cars on the origin edge, vehicles cannot be inserted into the network on their schedule. The departure delay of UE is more than twice than that of SO. From the data, although the route length is almost the same, SO has considerable advantages in terms of reducing trip duration, time loss, waiting time, and departure delay.

\subsection{Traffic distributions}

Figs. 5 and 6 show the variation of vehicle density with time along the alternative routes under UE and SO traffic assignment modes, respectively. As shown in the figure, the horizontal axis is the simulation time, starting from 0 seconds and lasting for 7 hours. The vertical axis shows the density of vehicles on each alternative path. From top to
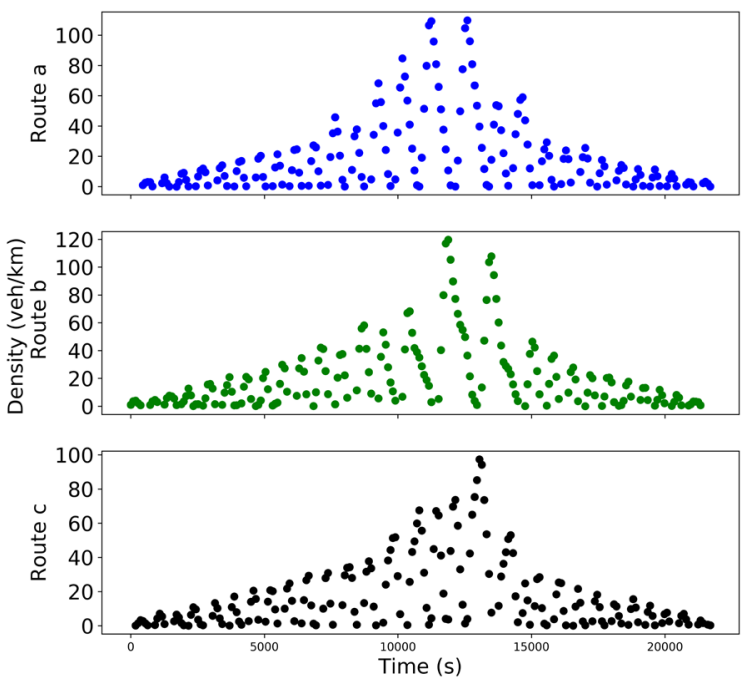

Fig. 5 Traffic distribution of SUE for the 3 routes of the network
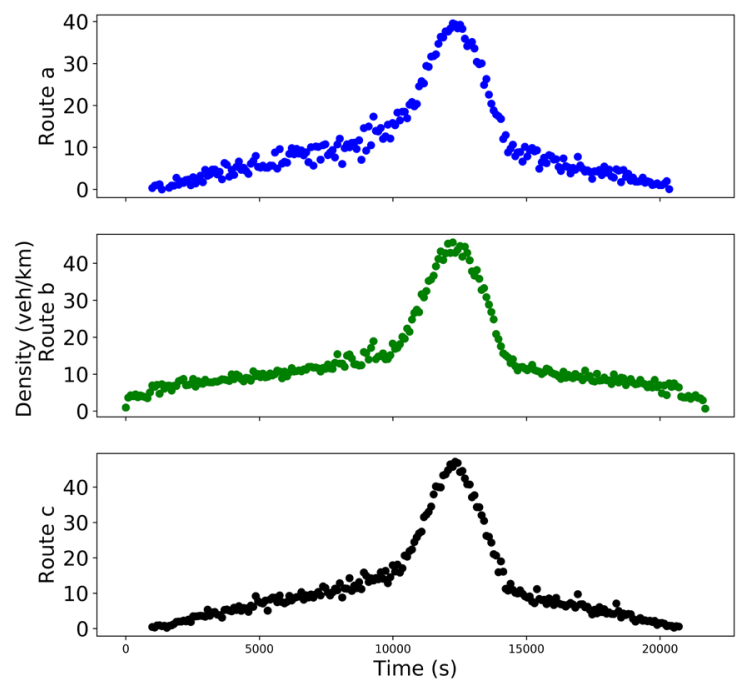

Fig. 6 Traffic distribution of DSO for the 3 routes of the network bottom, each of the subgraphs represents route a, route b, and route $\mathrm{c}$, respectively.

By comparing the two figures, one can easily find three main differences: the dispersion degree of density distribution, the arrival time of the peak density, and the highest density. The stochastic UE traffic assignment has a more dispersed vehicle density distribution. This assignment is less of a disadvantage when demand is small. Nevertheless, as travel demand approaches the capacity of the road network, one will find that some roads are overcrowded and others are underused. This phenomenon leads to drivers wasting more time on crowded streets, and underused roads are not abundantly utilized.

In the case of deterministic SO traffic assignment, the maximum vehicle density of each alternative path arrives almost simultaneously. However, in the stochastic UE traffic flow allocation, the vehicle peak density of each path is arriving one after another. When drivers choose a route based solely on travel time, it can lead to too many vehicles entering the same road in succession. As a result, roads become overcrowded. It takes a while for new drivers to realize that there is an underused road, and then for another wave of drivers to choose the underused road, which in turn causes the road to become overcrowded. It would take a considerable time before the new traffic would perceive another underutilized road and move en masse, leading to overcrowding.

The maximum density of stochastic UE allocation is more than twice than that of deterministic SO assignment, indicating that SO can significantly reduce or avoid excessive congestion. From the point of view of the peak vehicle density, the advantage of the high capacity of route $\mathrm{c}$ is exerted in the case of SO. Because travel demand is dynamic, the maximum density on route $\mathrm{c}$ is not that different from the maximum density on route $b$.

\section{Conclusion}

In this paper, two traffic assignment models have been investigated to explore the possible advantages of deterministic social optimum traffic assignment over the stochastic user equilibrium assignment. The newly introduced modified VDF was used to calculate travel time and marginal travel time. The system optimal assignment method of marginal travel time is realized by using the model. A simple fork road network was created using SUMO. In this model, two modes of traffic assignment were realized. By comparing the results of the two simulations, the possible benefits of deterministic SO were found. This study found that SO has considerable advantages in terms of reducing 
trip duration, time loss, waiting time, and departure delay under the same travel demand.

What is more, the stochastic UE traffic assignment has a more dispersed vehicle density distribution. Moreover, deterministic SO traffic assignment helps the maximum vehicle density of each alternative path arrive almost simultaneously. Last but not least, SO can significantly reduce or avoid the occurrence of excessive congestion.

The drawback of the present study is that the two traffic assignment models differ in two aspects: the certainty of

\section{References}

Beckmann, M., McGuire, C. B., Winsten, C. B., (1956) "Studies in the Economics of Transportation", Yale University Press, New Haven, CT, USA.

Daganzo, C. F. (1995) "Properties of link travel time functions under dynamic loads", Transportation Research Part B: Methodological, 29(2), pp. 95-98.

https://doi.org/10.1016/0191-2615(94)00026-V

Fukushima, M. (1984) "A modified Frank-Wolfe algorithm for solving the traffic assignment problem", Transportation Research Part B: Methodological, 18(2), pp. 169-177.

https://doi.org/10.1016/0191-2615(84)90029-8

Kucharski, R., Drabicki, A. (2017) "Estimating Macroscopic Volume Delay Functions with the Traffic Density Derived from Measured Speeds and Flows", Journal of Advanced Transportation, 2017, pp. 1-10.

https://doi.org/10.1155/2017/4629792

Lopez, P. A., Behrisch, M., Bieker-Walz, L., Erdmann, J., Flötteröd, Y. P., Hilbrich, R., Lücken, L., Rummel, J., Wagner, P., Wiessner, E. (2018) "Microscopic Traffic Simulation using SUMO", In: 21st International Conference on Intelligent Transportation Systems (ITSC), Maui, HI, USA, pp. 2575-2582. https://doi.org/10.1109/ITSC.2018.8569938

Patriksson, M. (2015) "The Traffic Assignment Problem: Models and Methods", Dover Publications, New York, NY, USA.

Pauer, G., Török, Á. (2019) "Static System Optimum of Linear Traffic Distribution Problem Assuming an Intelligent and Autonomous Transportation System", Periodica Polytechnica Transportation Engineering, 47(1), pp. 64-67.

https://doi.org/10.3311/PPtr.11548 choice and travel time calculation. It is unclear that which part exactly contributed to the advantage. The goal for the future is to examine the contribution of each factor.

\section{Acknowledgment}

This project (2019-2.1.7-ERA-NET-2021-00019) has received funding from the NRDI Fund by the National Research, Development and Innovation Office Hungary.

Pigou, A. (2013) "The Economics of Welfare", Palgrave Macmillan, London, UK.

Seger, M., Kisgyörgy, L. (2020) "Uncertainty Quantification of the Traffic Assignment Model", Periodica Polytechnica Civil Engineering, 64(4), pp. 1181-1201.

https://doi.org/10.3311/PPci.16396

Sheffi, Y. (1985) "Urban transportation networks: Equilibrium Analysis with Mathematical Programming Methods", Prentice-Hall, New Jersey, NJ, USA.

Sjoberg, K., Andres, P., Buburuzan, T., Brakemeier, A. (2017) "Cooperative Intelligent Transport Systems in Europe: Current Deployment Status and Outlook", IEEE Vehicular Technology Magazine, 12(2), pp. 89-97. https://doi.org/10.1109/MVT.2017.2670018

United States, Bureau of Public Roads (1964) "Traffic assignment manual: for application with a large, high speed computer", U.S. Department of Commerce, Bureau of Public Roads, Office of Planning, Urban Planning Division, U.S. Government Printing Office, Washington, DC, USA.

Wardrop, J. G. (1952) "Road Paper. Some Theoretical Aspects of Road Traffic Research", Proceedings of the Institute of Civil Engineers, 1(3), pp. 325-362.

https://doi.org/10.1680/ipeds.1952.11259 